\title{
Spectrometry: Photon sorting at the speed of light
}

\author{
H. J. P. Vink ${ }^{*}$, J. P. R. Day, J. B. C. G. Volatier, M. D. Nijkerk \\ TNO Technical Sciences, Optics Department, Stieltjesweg 1, 2628CK, Delft, The Netherlands
}

\begin{abstract}
The fact that every spectrometer can sort light by wavelength at the speed of light is intriguing. The field of spectrometry is a long-existing and ever-changing one. The application areas extend from optical communication to possible extraterrestrial life detection, health monitoring, environmental monitoring and quite a long list of other topics. TNO has played a role in several of these areas, always using state of the art designs and components. Some of the recent developments are described, as well as a possible path for (near) future developments.
\end{abstract}

Any spectrometer consists of a telescope, slit, collimator, disperser and an imager. Each of these functions is discussed using and even pushing progress in the manufacturing and design capabilities of the industry.

The progress from a two-mirror spherical telescope for a pushbroom space-based daily global coverage spectroscopy instrument OMI to a two-mirror freeform telescope for TROPOMI is described, the design and manufacturing of supergratings showing very little straylight, freeform mirrors and the use of deliberately decentered lenses is shown. A nearfuture small-satellite system is shown that is being built and tested as this paper was written.

Keywords: Optical design, spectroscopy, super-gratings, straylight, free-form mirror, small-satellite system

\section{INTRODUCTION}

Space-based earth observation spectrometers are used extensively for environmental monitoring of gas and aerosol concentrations in the earth's atmosphere. They deliver essential information on the levels of numerous anthropogenic and natural gases and aerosols, including $\mathrm{O}_{3}, \mathrm{NO}_{2}, \mathrm{SO}_{2}, \mathrm{CO}$ and $\mathrm{CH}_{4}$, with exceptional accuracy and coverage. As a result they are a critical sensor for the monitoring of climate change and pollution on a global scale. Extremely stringent performance requirements and atypical environments drive the optomechanical design of these instruments repeatedly beyond the state-of-the-art with each new generation, with concomitant benefits for earth-based instruments. In this paper we describe the optical design of earth observation spectrometers with particular focus on TROPOMI.

Earth observation satellites operate in nearly polar orbits at an altitude of $\sim 800 \mathrm{~km}$. A large instantaneous field-of-view (IFOV) coupled to an orbital period of $\sim 100$ minutes lead to daily full earth coverage. This large IFOV requirement means that most earth observation spectrometers use the "pushbroom" technique. A pushbroom imaging spectrometer is characterized by a large IFOV in the across-track or swath direction, and a small IFOV in the along-track direction. The swath direction is imaged by the telescope and spectrometer optics to give one spatial direction and the motion of the satellite serves to generate the second spatial direction. Inside the spectrometer, light is dispersed in the along-track direction to give the spectral direction. The impossibility of imaging three dimensions (two spatial and one spectral) onto a two dimensional detector is circumvented by the use of time as the third dimension and scanning in the second spatial direction. Other approaches such as active scanning and filter based imaging have also been adopted, but are not discussed further in this paper.

TNO in The Netherlands has a long history in the design and manufacture of space-based spectrometers. Most recently, the precursor instrument TROPOMI ${ }^{1,5}$ (TROPOspheric Monitoring Instrument) for the upcoming Sentinel5 program was designed and manufactured to be launched in 2016. TROPOMI builds on the heritage developed at TNO; in particular the opto-mechanical design of the pushbroom spectrometer OMI (Ozone Monitoring Instrument ${ }^{2,3}$ ) launched in 2004 on the NASA Aura satellite. The optical design principle of OMI was used for TROPOMI, however several requirements were tightened considerably for this next generation instrument. The requirements with most impact upon the optical design were the change of spatial resolution, which tightened from $24 \times 13 \mathrm{~km}$ to $7 \times 7 \mathrm{~km}$, and more stringent signal-tonoise levels, affecting in particular the straylight performance of the instrument. These changes led to the use of several disruptive technologies. 
The tightened requirement on spatial resolution led to the use of freeform mirrors for the telescope, which were manufactured and measured at the optical workshop of TNO in Delft. The design and manufacturing of these freeform mirrors is nontrivial, and they are discussed in more detail below

The improved straylight performance necessitated improvements in grating technology. TROPOMI contains a shortwave infrared (SWIR) wavelength channel, based on a silicon-immersed grating. ${ }^{4}$ This grating manufacturing technology was taken as the basis for the manufacturing of reflective "super-gratings". These revolutionary components exhibit straylight levels potentially far superior to classically manufactured ruled or holographic gratings. They are planned to be used in future spectrometer instruments which have very stringent straylight requirements.

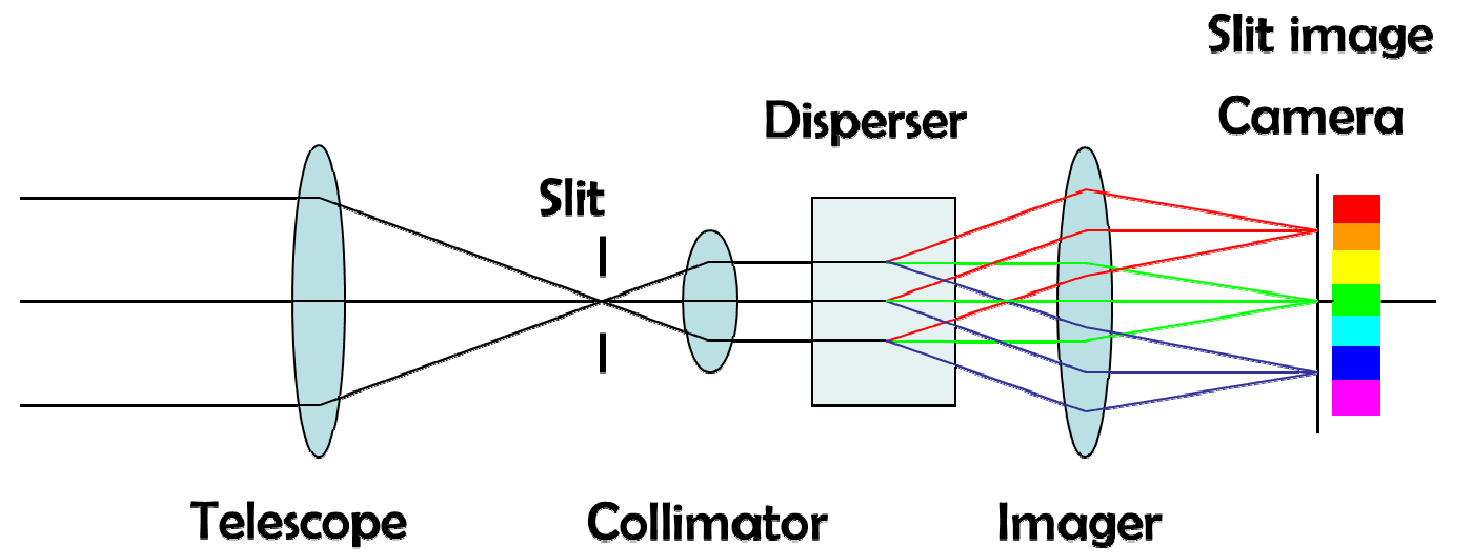

Figure 1. Paraxial schematic of a spectrometer.

A typical earth-observation spectrometer consists of a telescope, slit, collimator, dispersive element, imager and detector, as sketched in Figure 1. These functional blocks will be exemplified by TROPOMI, with particular emphasis on the developments to reach the required performance levels. The remainder of this paper is presented as follows. In Section 2 we present the TROPOMI telescope with particular emphasis on the design and tolerancing of freeform mirrors and the polarization scrambler. In Section 3 we discuss the UV1 channel spectrometer of TROPOMI with emphasis on supergratings, imager design and a graded coating for straylight rejection. Finally in Section 4 we describe a smallsatellite mission that could be employed in the near future.

\section{TELESCOPE}

The telescope in an imaging earth-observation spectrometer images a strip from the earth's surface onto the slit. The field-of-view (FoV) is large in the swath or across-flight direction and small in the perpendicular direction (along flight direction). The FoVs for TROPOMI are given in Table 1.

Table 1: Selected properties of TROPOMI

\begin{tabular}{|l|l|l|}
\hline \multicolumn{1}{|c|}{ Property } & \multicolumn{1}{c|}{ Unit } & \multicolumn{1}{c|}{ Value } \\
\hline Swath FoV (at entrance pupil) & degrees & 108 \\
\hline Swath FoV (at earth) & $\mathrm{km}$ & 2600 \\
\hline $\begin{array}{l}\text { Along track FoV (at entrance } \\
\text { pupil }\end{array}$ & degrees & 0.5 \\
\hline Along track FoV (at earth) & $\mathrm{km}$ & 7 \\
\hline f/\# (swath) & - & 9 \\
\hline f/\# (along-track) & - & 10 \\
\hline Wavelength range & $\mathrm{nm}$ & $270-2385$ \\
\hline
\end{tabular}


The TROPOMI telescope is a two-mirror, reflective, telecentric, f $\theta$ telescope, shown in Figure 2 and Figure 3. TROPOMI itself is shown in Figure 4. The telescope delivers the input scene to four separate spectrometers spanning from $270-2385 \mathrm{~nm}$ and therefore a reflective two-mirror design was chosen due to the wide bandwidth. Telecentricity is required to simplify the coating design for the dichroic mirrors that divide the light over the four spectrometers.

The two telescope mirrors are referred to as primary (mirror) and secondary (mirror), in the order in which light from the earth surface passes through the telescope. In the OMI instrument, these mirrors were both spherical. However, the more stringent requirements on spatial resolution necessitated the use of freeform surfaces without spherical or axial symmetry. These freeforms are described in more detail below.

The primary mirror creates an astigmatic intermediate image close to the primary. A field stop in the swath direction is positioned at the across-track focus. This field stop is oversized in the along-track direction. A baffle is also placed at the along-track focus to aid in the removal of stray light, however the field stop in the along-track direction is the spectrometer slit. The intermediate image is re-imaged by the secondary on the telescope focal plane. Simultaneously, the entrance pupil is imaged to infinity, which is equivalent to the condition of telecentricity.

The telescope has different f-numbers in the two perpendicular directions. This difference has been achieved by dimensioning the aperture stop (pupil) to a rectangular shape. The telescope furthermore has different focal lengths in the flight and swath directions (anamorphotic), achieved by making the radii of curvature of the two mirrors in the two perpendicular directions substantially different.

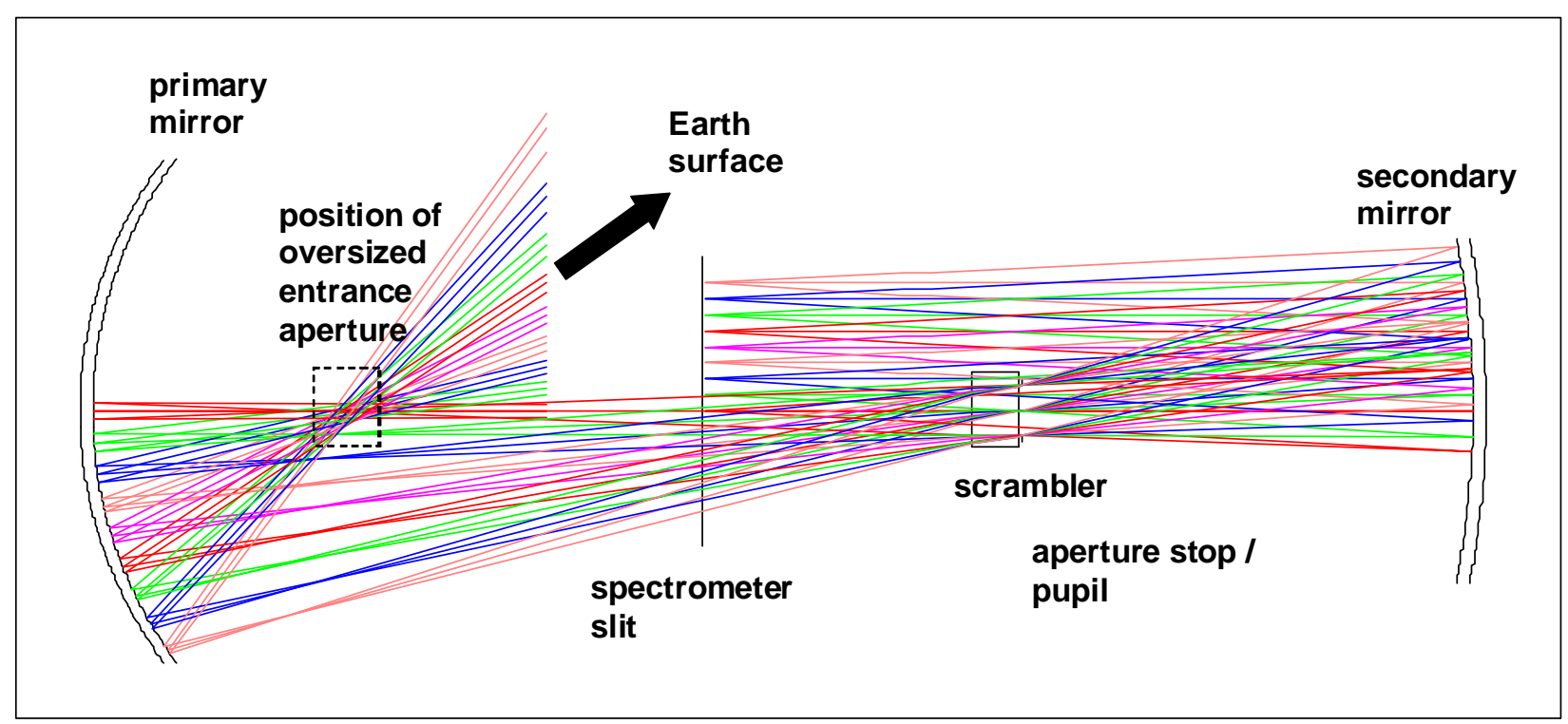

Figure 2. Telescope cross-section along swath direction. One half of the field is drawn. 


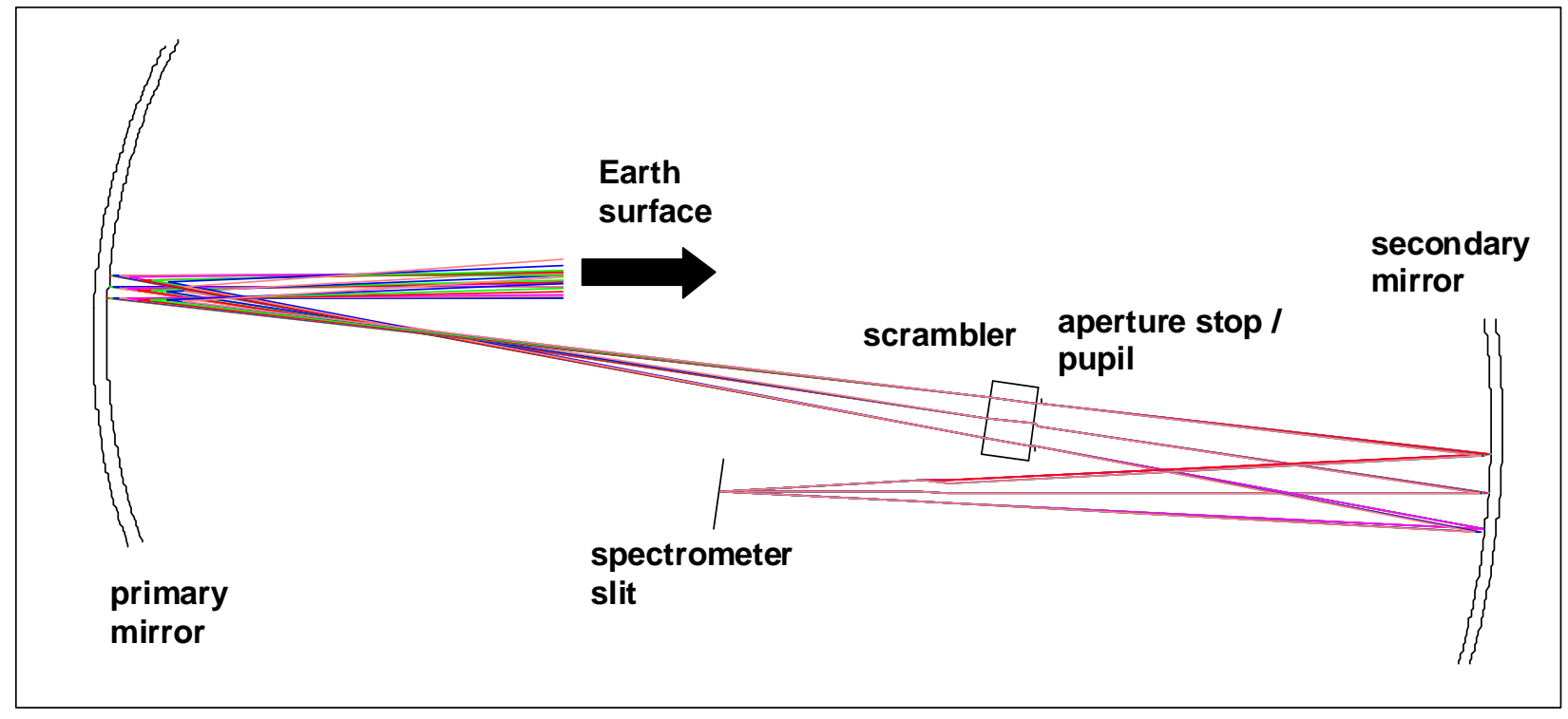

Figure 3. Telescope cross-section along flight direction

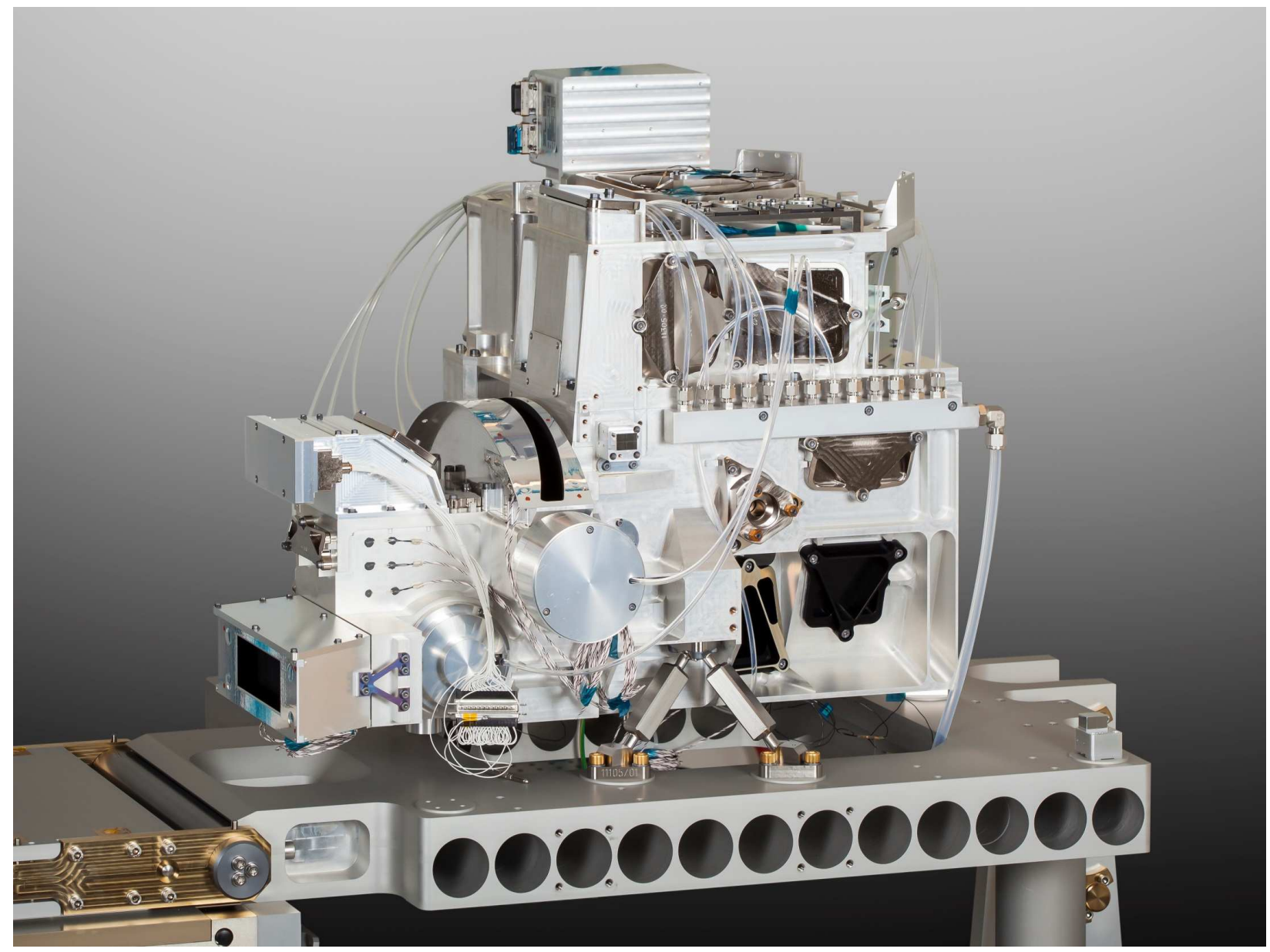

Figure 4. TROPOMI flight model. 


\section{Freeform mirrors}

The telescope consists of two freeform mirrors. These freeform mirrors are unconventional surfaces that are fabricated in an unconventional manner (i.e. they are not surfaces of revolution). As a result, tolerancing freeforms is an extensive task, in which the impact of surface waviness and midspatials must be investigated.

The mirrors were fabricated using the slow servo method on a diamond turning machine. The work piece was mounted on a rotary stage with the diamond chisel cutting out the surface by moving in the z-direction and the r-direction as the work piece rotates about the $\mathrm{z}$-axis. On the basis of symmetry it can be reasoned that the dominant surface sag error will be rotationally symmetric. The source of the surface sag error is typically long term thermal instability of the chisel. The grooves of the chisel do not lead to shape error since the grooves are completely removed by a post polishing step.

This expected shape of the surface sag error has been confirmed in the mirror bread board program. In Figure 5, a scan of the surface sag error along the swath direction of the M1 mirror from the bread board program is shown. The 2D surface sag error function is substantially rotationally symmetric.

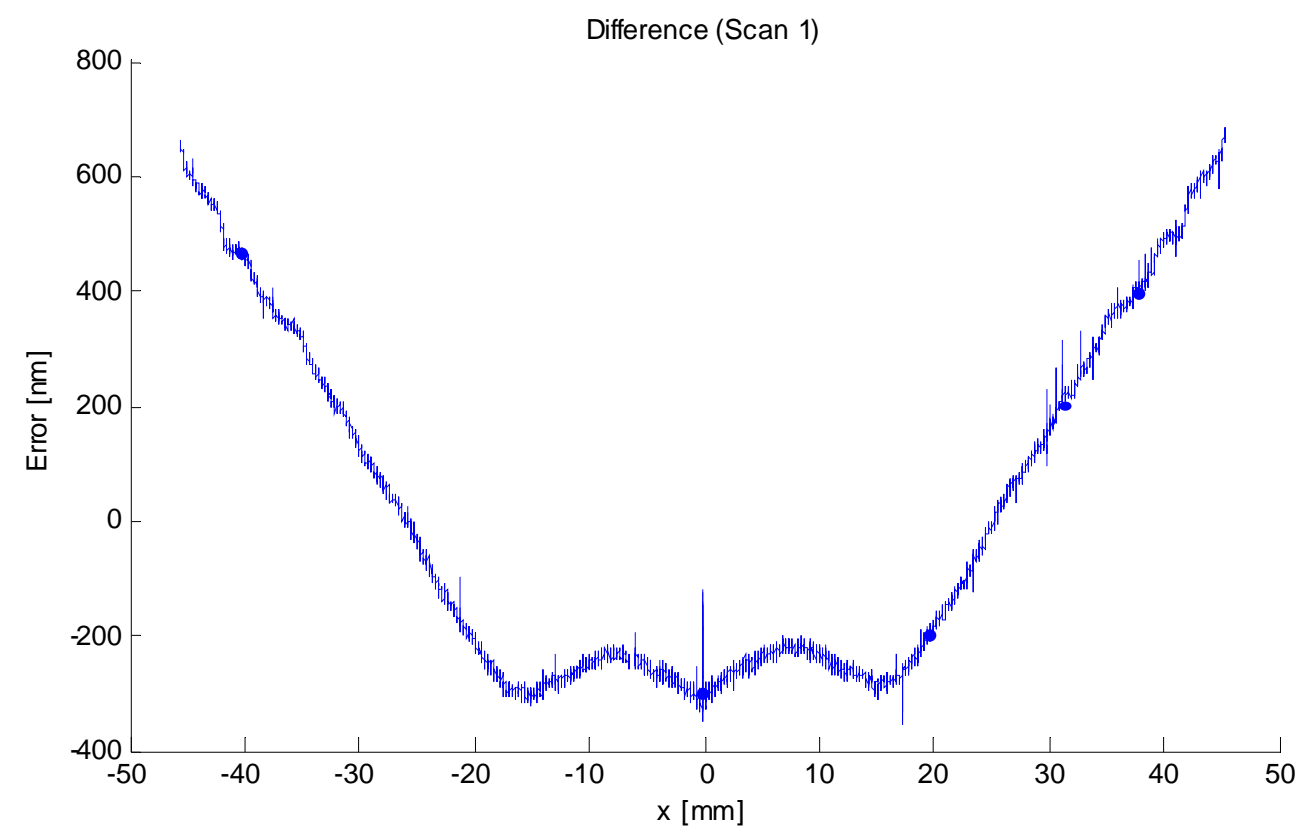

Figure 5. 1D scan of the 2D surface sag error function of M1 from the bread board program.

Surface sag errors can be modelled as a one-dimensional sag error that is rotated about the axis of revolution. The onedimensional sag error can be practically any sequence of ripples with arbitrary slope. This makes the surface sag error fundamentally different from other errors; e.g. lateral misalignment in one dimension can be described in terms of a onedimensional statistical distribution, whereas a general surface sag error corresponds to an infinite-dimensional statistical distribution, each dimension corresponding to a linearly independent generic sag function. For simplicity, the analysis here is restricted to cosine-shaped sag errors, but even within this restriction the statistical distribution is essentially infinite-dimensional. For a systematic analysis, the cosine period and amplitude is varied. Thus, the surface sag error, $\Delta$ Sag, can be written:

$\Delta \operatorname{Sag}(r)=\operatorname{Acos}(2 \pi r /$ period $)$

with $r$ the radial coordinate with respect to the center of rotation of the mirror as it is cut out in the slow servo process. This point coincides with the vertex of the mirror (more exactly: the vertex of the freeform's base sphere). An example of such a midspatial surface error is shown in Figure 6.

Surface errors related to the freeform fabrication process (diamond turning) that are taken into account are a concentric ripple over the full surface. Thus the sag function of the mirror surfaces may include a cosine-shaped radially concentric 
ripple of arbitrary, user defined, amplitude and period, to facilitate modelling of the ripples due to slow-servo diamond turning.

Note the jargon here: the term irregular surface error is reserved for cylindrical shaped surface errors, i.e. surface sag errors shaped like the letter "U". More complex errors with more oscillations about the nominal shape are referred to as midspatial surface errors.

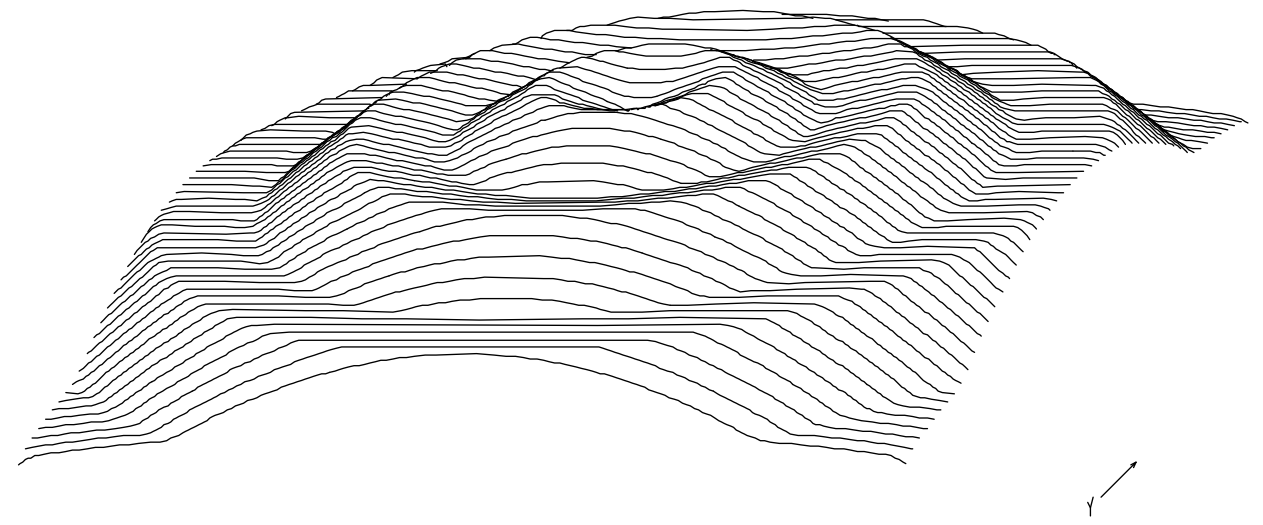

Figure 6. Example of midspatial surface error due to slow-servo diamond turning. The period is exaggerated for demonstrative purposes.

\section{Polarization Scrambler}

Earth observation instruments have stringent requirements on polarization sensitivity. A polarization scrambler is therefore situated at the aperture stop in between the two mirrors. The polarization scrambler is a Dual Babinet Compensator Pseudo depolarizer (DBCP), which consists of two pairs of birefringent wedges as shown in Figure 7. Its operating principle is discussed below.

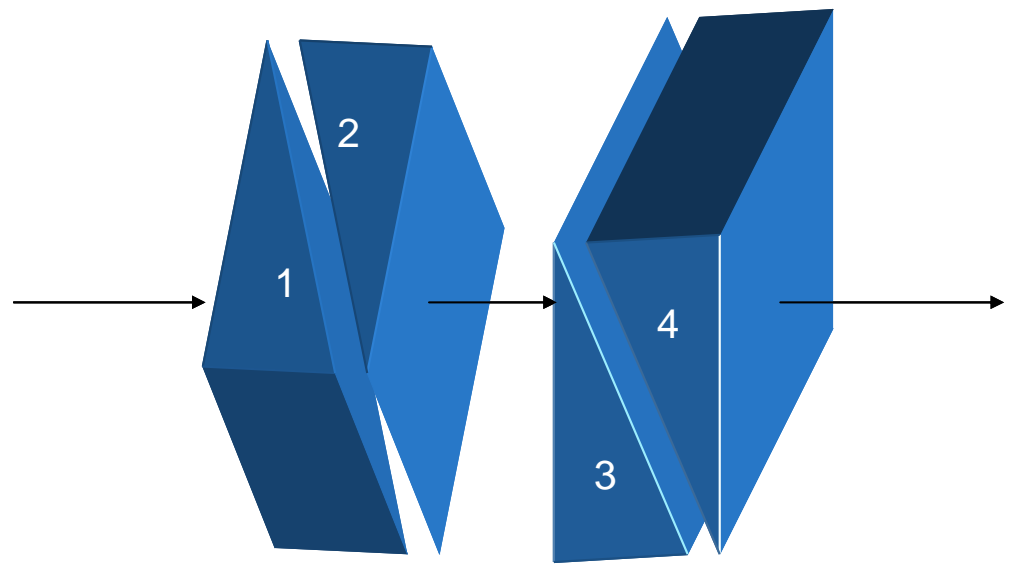

Figure 7. Schematic 3D layout of the scrambler, which shows how the scrambler is built from two wedge pairs. The arrows indicate the direction of light propagation.

A birefringent crystal positioned perpendicular to a beam of light, with its crystal optic axis in the plane of the plate (i.e. perpendicular to the light) causes a change of phase of the incident light. The incident electric field is decomposed in two components: one oriented along the crystal optic axis, and one perpendicular to it. Due to the difference in refractive index of the two orientations, one component experiences a phase retardation with respect to the other component, which results in a phase change of the total electric field. The phase retardation depends on the optical path length in the crystal. Consequently, as the plate thickness varies over the beam, the phase change varies. Thus, when fully polarised light passes a birefringent wedge, the exiting light contains many polarization states. A single wedge thus acts as a polarization scrambler. 
A second effect of birefringence is that the ordinary and extraordinary ray experience a different angle of refraction, which has the consequence that a single ray is generally split into two rays after passing through a birefringent wedge. One wedge acts as a prism, causing an undesired angular deflection. Therefore, the wedge is matched with another wedge that has an opposing wedge angle. To ensure that the depolarizing effect of the first wedge is not undone by the second wedge, the crystal optic axes must be oriented mutually perpendicular.

Incident light that is polarized exactly along the direction of the crystal axis of one of the two wedges passes the wedge pair without a phase change. To take care of depolarization of this light, a second wedge pair is needed that has crystal optic axis orientation at $45^{\circ}$ with respect to the first wedge pair.

This assembly of four wedges is the DBCP. Each wedge splits an incident ray into an ordinary and extraordinary ray, so the DBCP turns a single ray into sixteen rays. This effect is referred to as the diamond effect after the shape of the envelope of these sixteen rays in angular space. The bulk of the energy is typically contained in four of the sixteen rays that form the corners of the diamond. The diamond effect is an unwanted side effect leading to a degradation of spot size.

The DBCP delivers good scrambling performance, but has an additional drawback. The diamond barycentre moves up and down with orientation of linear polarization, which leads to polarization-dependent pointing through the telescope and thus coregistration error. To counter the coregistration error, the first wedge pair is split in the across track direction, as shown in Figure 8. This split duplicates the diamond, creating a neighbour shifted in the along-track direction for each of the four spots. This causes a further degradation of spot size in the along-track direction but it also comes with a benefit: as the orientation of the incident linear polarization rotates, the barycentre of the different diamonds move in opposite directions, thereby cancelling the polarization-dependent pointing.

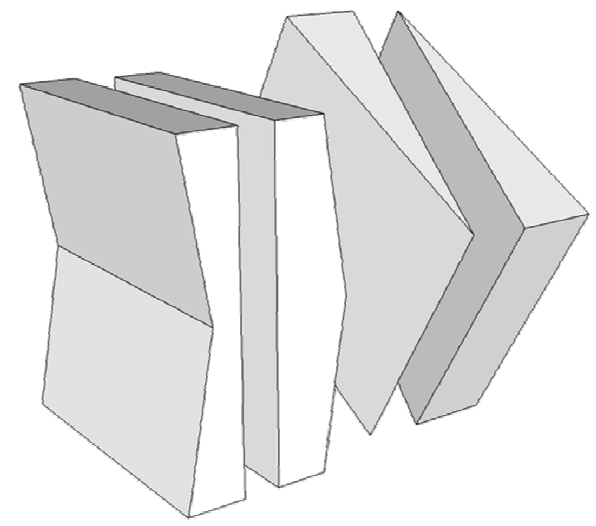

Figure 8. DBCP variant with split aperture

\section{SPECTROMETER}

We illustrate the performance of an earth observation spectrometer with the UV1 channel from TROPOMI. Figure 9 shows the spectrometer optics for the UV1 channel from slit to detection plane as modeled in Zemax. The view shown is the spectral direction and the rays are colored by wavelength. The beam from the telescope illuminates a reflective slit telecentrically. This slit is not yet the limiting slit for the spectrometer. This slit is re-imaged by use of optics CL1 and FM1 to the entrance slit of the spectrometer channel, which is just in front of CL2. The dichroic shown splits the SWIR wavelength band from the UV1 band. FM2-FM5 are fold mirrors that are required due to the limited envelope within the TROPOMI instrument. The collimator lens CL3 images the pupil onto the grating. FM6 folds the light towards the imager, consisting of three fused silica lenses, which form the image on the detector. We now consider various aspects of the spectrometer in more detail. 


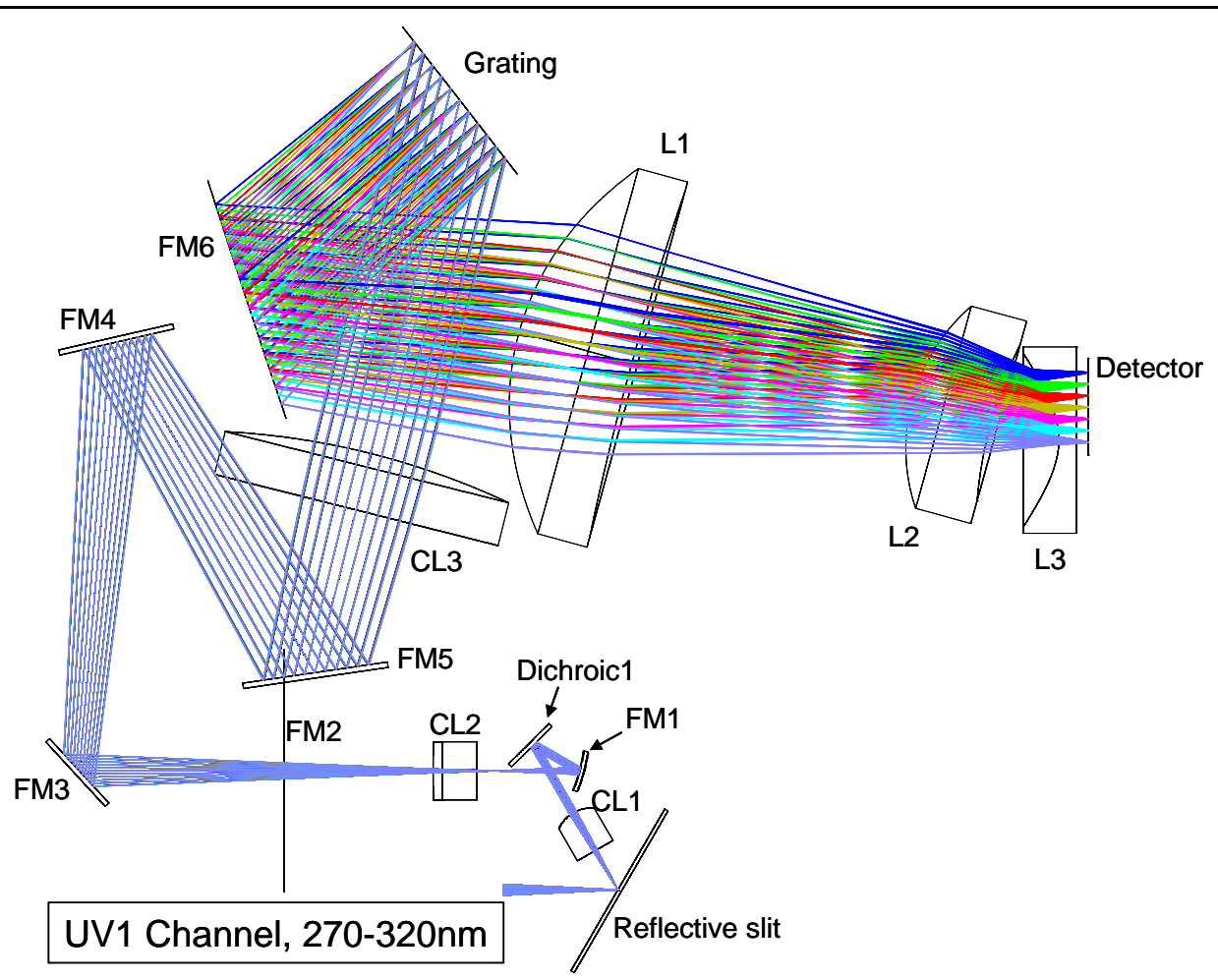

Figure 9. Layout of the UV1 channel optics from slit to detection plane. Plane of view includes spectral direction. Different color corresponds to different wavelength

\section{Supergratings}

The scattering properties of the grating is often the limiting factor in the straylight performance of a spectrometer. Conventional gratings were deemed insufficient for future spectrometer systems that would need even lower straylight performance, leading to the development of "supergratings" (patent pending). The properties of these supergratings are potentially far superior to classically manufactured ruled or holographic gratings.

For TROPOMI, an immersed grating was developed by SRON and TNO for the SWIR channel. ${ }^{4}$ This grating is based on wet-etching of crystalline silicon. The possibility of replicating (and thus inverting) the groove pattern of this immersed silicon grating with a grating replication process enables the manufacturing of a reflective non-immersed grating. SEM pictures of $\langle 100\rangle$ and a $\langle 112\rangle$-etched silicon substrate are shown in Figure 10.

The etching of silicon can result in very low roughness and in a very predictable groove profile. The low roughness results in low scattering, which corresponds to low straylight. The predictable groove profile results in a predictable efficiency of the grating. The efficiency prediction from theory of these gratings can match the measured efficiency within a few percent, which means that the optical designer needs less margin for overall throughput which leads to a smaller design. For space instruments, both low straylight and a lower volume and mass are very much appreciated. 


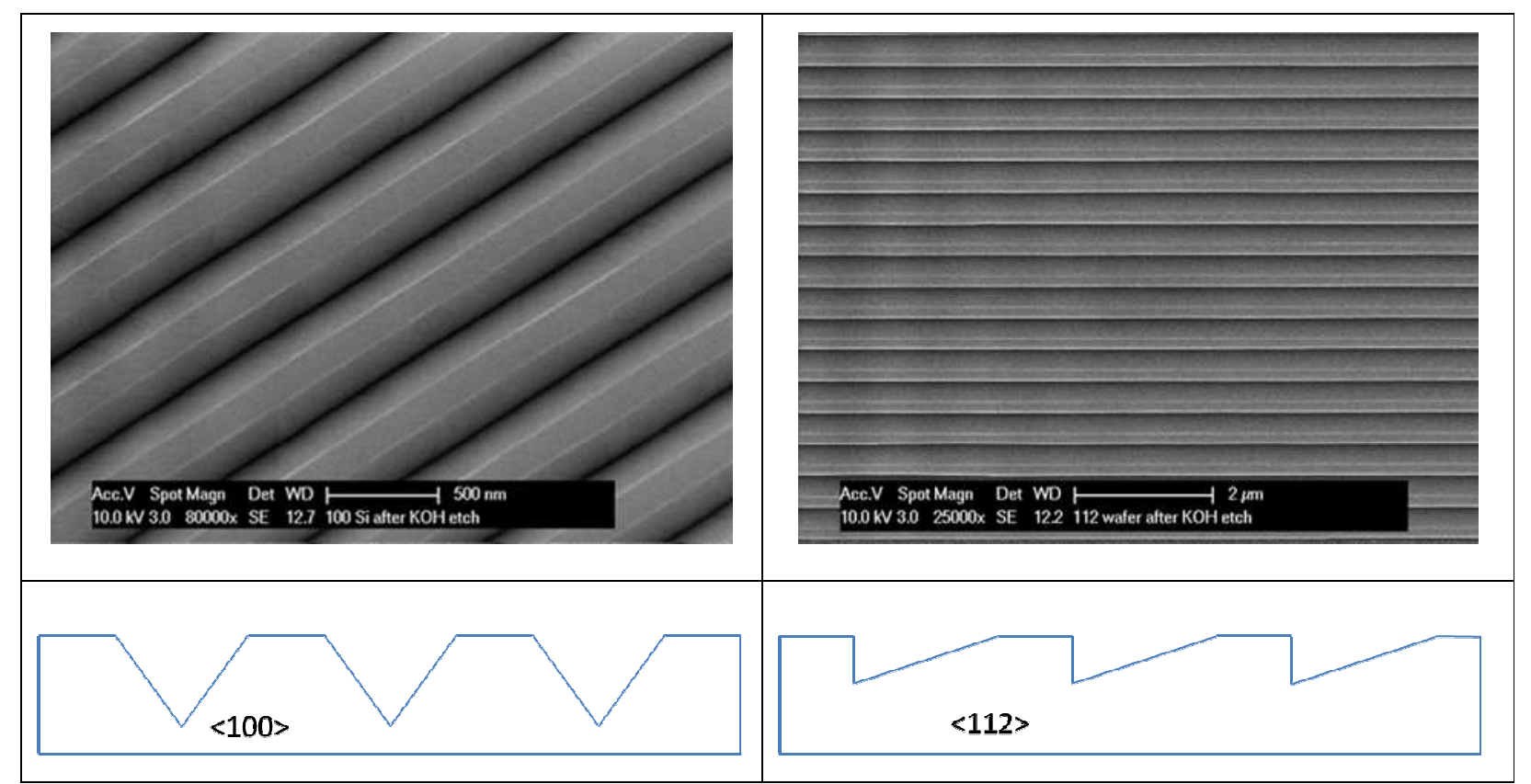

Figure 10. SEM picture of $\langle 100\rangle$ (top left) and $\langle 112\rangle$ (top right) silicon crystal orientation. The groove profile is given below the SEM pictures

As for any component, there are limitations in both the design and the performance of this type of grating. The groove profile is fixed by the crystal structure of silicon. The blaze angle can be changed, but the included angle cannot. A single groove consists of a horizontal and an angled part. The ratio of their widths is a design parameter. This leads to the following design parameters:

- Groove density: Few lines per millimeter to >2500 lines per millimeter

- Blaze angle: Any, although there are a few "standard" crystal orientations available like $<112>$ which results in a $90^{\circ}$ groove edge

- $\quad$ Ratio of horizontal to angled width

- Wavelength range: Depends on applied coating

Since the grooves should be aligned to the crystal structure, it is impossible to manufacture curved grooves with low roughness. A modest curvature of the grating surface (either convex or concave) should be possible, although this has not been tested yet.

In principle, this manufacturing method leads to grooves that each consist of crystal planes. The expected roughness of a perfect crystal plane is near zero. Measurements on a $\langle 100\rangle$ silicon wafer show BSDF levels that are orders of magnitude lower than the BSDF of a 0.7-nm RMS roughness mirror as shown in Figure 11. It might be possible to generate a zero-roughness grating, however particulate and molecular contamination will cause scattering of light as well. If the total scattering of a surface is dominated by contamination, there is no gain in using a surface with lower roughness. Nevertheless, improvement to roughness levels below 1-nm RMS will result in significant straylight improvement.

The BSDF of several gratings has been measured at TNO. As a reference a polished mirror and a silicon flat wafer were also measured. All measurements were performed using a laser operating at $532 \mathrm{~nm}$. 


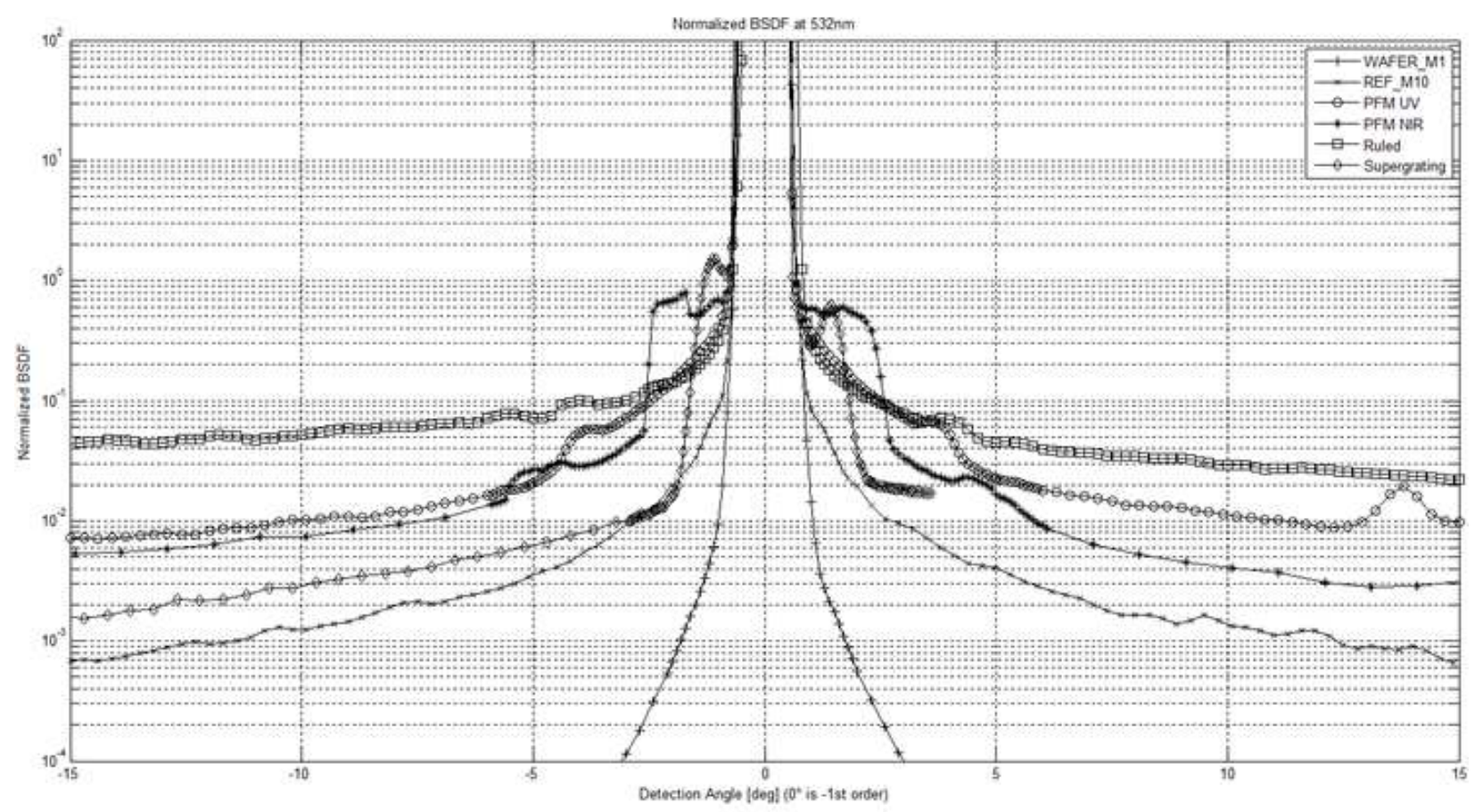

Figure 11. Measured BSDF of four different gratings and two reference mirrors

The BSDF in units $1 / \mathrm{sr}$ is given in Figure 11 for an angular range of \pm 15 degrees around the first diffraction order for each grating and around the specular reflection for the two reference mirrors. The "WAFER_M1" curve corresponds to a flat wafer, this is by far the lowest BSDF measured. In theory, the supergrating could be at this level as well. The curve corresponding to "REF_M10" was measured for a 0.7-nm RMS roughness mirror. The two curves "PFM UV" and "PFM NIR" are measurements on space qualified gratings for the UV and NIR wavelength bands. The UV grating is a commercially available grating, the NIR grating was developed by Carl Zeiss ${ }^{4}$ for the NIR wavelength range. A typical triangular sawtooth groove-profile would lead to insufficient polarization performance of this NIR grating. The squares and worst performing grating in this set of measurements is a ruled grating. This particular ruled grating was a commercially available replicated grating.

The supergrating is marked by diamonds and shows the lowest BSDF values for angles larger than $2^{\circ}$. This grating is one of the first set of replicas that was manufactured and already showed excellent straylight performance.

\section{Imager}

The imager in the UV1 spectrometer consists of three fused silica lenses. All lenses are decentered and tilted with respect to the optical axis in order to get a good distortion performance, the nominal performance is free of distortion to the micron level. The last lens together with the CCD-plane is tilted, which corrects to axial color aberration. This combination of decentered and tilted optics and detector therefore deliver good achromatic performance with only one lens material. This use of one lens material is particularly relevant in space applications as it greatly improves the thermal properties and predictability of the spectrometer and the ease of mounting. On the last surface, a graded coating was designed, this is more detailed in the next section.

\section{Graded coating}

In the UV1 channel, the spectral radiance of earth varies by three orders of magnitude from 270 to $310 \mathrm{~nm}$ as shown in Figure 12. It is expected therefore that straylight from the longer wavelengths will affect the performance in the lower wavelength range if no corrective measures are taken. In TROPOMI, a spatially varying shortpass coating is used on the planar face of L3. For example, at the nominal location of 270-nm light on L3, the coating transmits 270-nm light and reflects wavelengths $>285 \mathrm{~nm}$. This $15-\mathrm{nm}$ difference in the nominal light and cut-on wavelength is constant across the surface of L3, although the cut-on wavelength itself is not constant. This graded coating delivered superior straylight performance to an uncoated equivalent. 


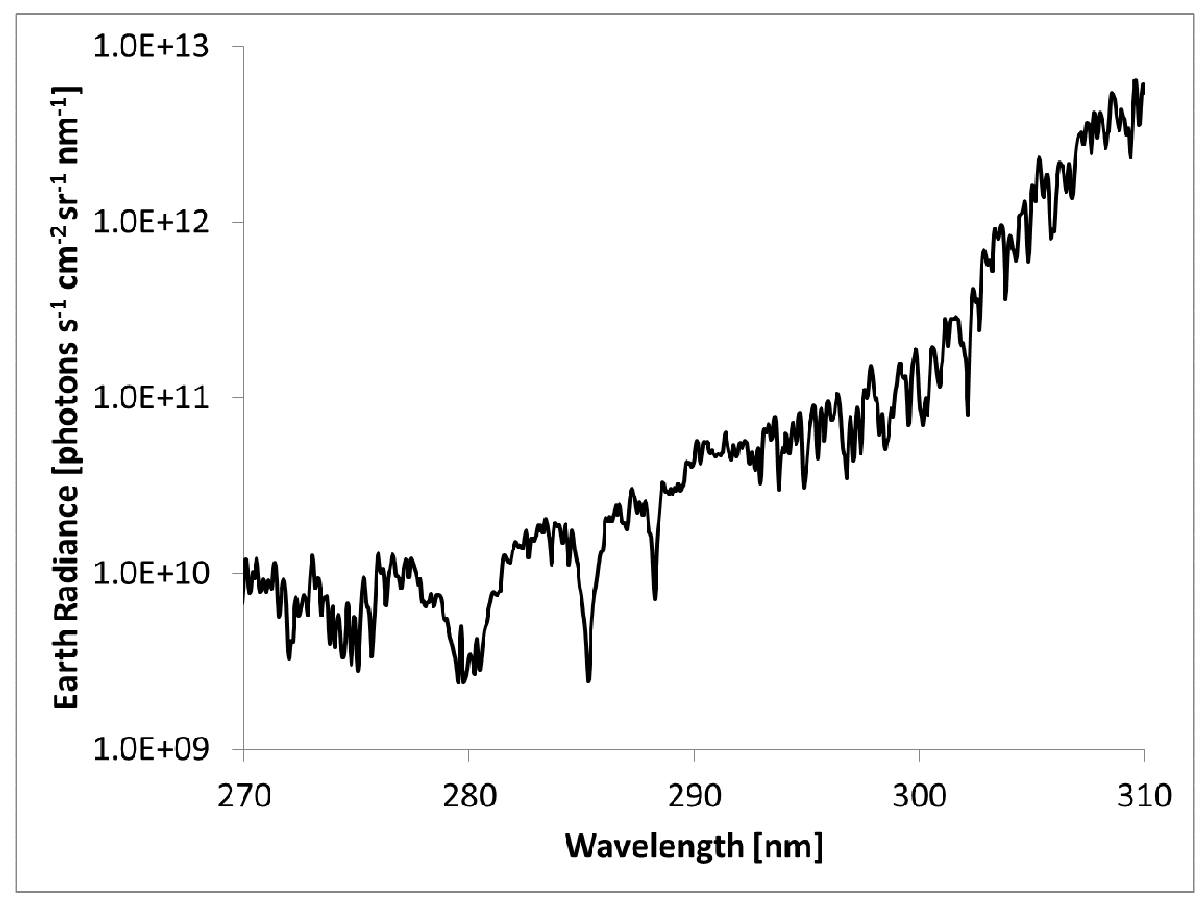

Figure 12. Earth radiance in UV1 channel.

A photograph of the manufactured component using white light illumination in reflection is shown in Figure 13.

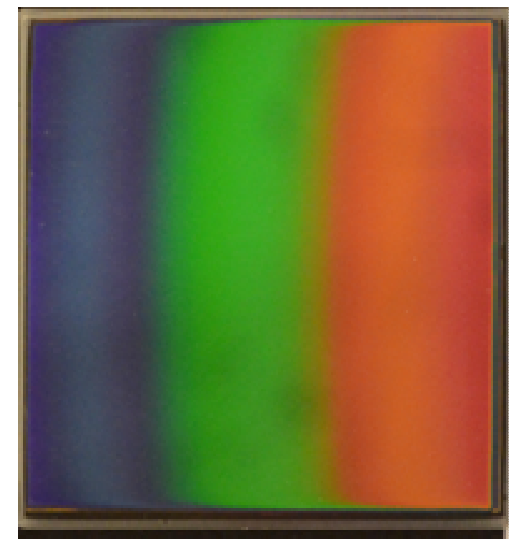

Figure 13: Photograph of graded coating on the component

\section{4. (NEAR) FUTURE SPECTROMETER DESIGNS}

Until recently the Earth Observation (EO) market has been dominated by institutionally driven satellites, which are big, heavy and expensive. Although these institutional missions will continue to exist, a more 'commercially' oriented EO market is starting to emerge, targeting specific data products, such as Nitrogen dioxide (NO2), Sulfur dioxide (SO2) and aerosols. A small-satellite mission was defined to provide local, region and national governments with cost-effective $\mathrm{NO} 2$ pollution level data at high spatial resolution. An orbital analysis shows that by employing orbits with a $60^{\circ}$ inclination the number of daily observations can be increased up to a factor of two, compared to the conventional sunsynchronous orbits. Furthermore, as the local observation time shifts over the course of days and weeks, $60^{\circ}$ inclined orbits avoid 'blind' time frames, in which no observations are made.

TNO has designed an instrument that fits within the dimensions of a standardized satellite platform made by Innovative Solutions in Space (ISIS). Two separate instruments, possibly aiming at two different data products, can be fitted within 
a single small-satellite platform. The instrument design is extremely compact because of the application of a range of new technologies (most notably free-form mirrors and lost-wax casting with 3D printed moulds). Components for an instrument demonstrator have successfully been produced and are being assembled in the summer of 2015.
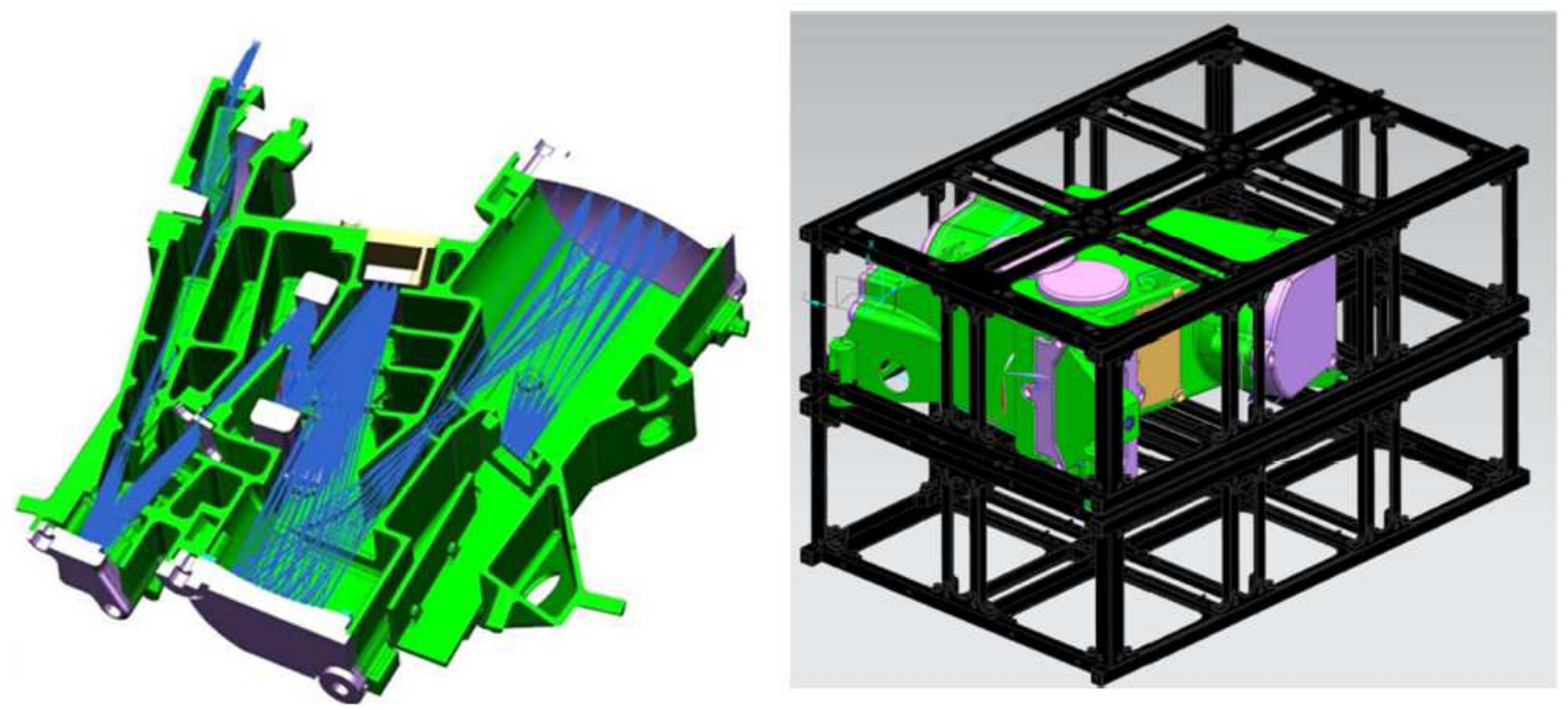

Figure 14: Cross section (left) of the designed imaging spectrometer and schematic (right) showing how this spectrometer fits into the standardized structure of the satellite.

In Figure 14 the spectrometer is shown inside the standardized structure of the satellite. The opto-mechanics fit a volume of $10 \times 20 \times 20 \mathrm{~cm}$. Two of these units (two wavelength bands) in a volume of $20 \times 20 \times 30 \mathrm{~cm}$ leaves sufficient room for the supporting hardware like electronics, communication, attitude and thermal control.

\section{ACKNOWLEDGEMENTS}

The reported work on TROPOMI was made possible by the Netherlands Space Office (NSO) in cooperation with ESA.

TROPOMI has been developed in a cooperation between the principal investigators KNMI and SRON and an industrial team led by Airbus Defence and Space Netherlands.

TNO was subcontracted to perform the opto-mechanical design, performance analyses, integration and alignment of the UV, UVIS and NIR channels, telescope and calibration unit of TROPOMI.

Airbus Defence and Space Netherlands has been responsible for the mechanical, thermal and electrical integration, environmental testing and for the calibration of TROPOMI.

The immersed gratings for the SWIR channel of TROPOMI were manufactured by the Netherlands Institute for Space Research (SRON). 


\section{REFERENCES}

[1] Veefkind, P., Kleipool, Q., Aben, I. and Levelt, P., "The TROPOspheric Monitoring Instrument (TROPOMI)" Geophysical Research Abstracts 17, (2015).

[2] Levelt, P. F., van den Oord, G. H. J., Dobber, M. R., Malkki, A., Visser, H., de Vries, J., Stammes, P., Lundell, J. O. V. and Saari, H., "The ozone monitoring instrument", IEEE Transactions on Geoscience and Remote Sensing 44(5), 1093-1101 (2006)

[3] Dobber, M. R., Dirksen, R. J., Levelt, P. F., van den Oord, G. H. J., Voors, R. H. M., Kleipool, Q., Jaross, G., Kowalewski, M., Hilsenrath, E. Leppelmeier, G. W., de Vries, J., Dierssen, W and Rozemeijer, N. C. "Ozone monitoring instrument calibration", IEEE Transactions on Geoscience and Remote Sensing 44(5), 1209-1238 (2006)

[4] Steiner, R., Pesch, A., Erdmann, L. H., Burkhardt, M., Gatto, A., Wipf, R., Diehl, T., Vink, H. J. P, van den Bosch, B. G. "Fabrication of low straylight holographic gratings for space applications," Proc. SPIE 8870, 17 (2013)

[5] Doornink, J. et al., "The TROPOMI instrument, last steps towards final integration and testing,", Proceedings of the International Conference on Space Optics (2014)

[6] Innovative Solutions In Space (ISIS), <http://www.isispace.nl>, www.isispace.nl/ 